International Journal of Engineering \& Technology, $7(1.1)(2018)$ 306-310
International Journal of Engineering \& Technology
Website: www.sciencepubco.com/index.php/IJET
Research paper

\title{
Load balancing in cloud computing
}

\author{
Sudhir Kumar M ${ }^{1}$, P Chakravarthi ${ }^{1 *}$, B Jagadeesh ${ }^{1}$, M sai prakash ${ }^{1}$ \\ ${ }^{1}$ Computer Science and Engineering, Koneru Lakshmaiah Educational Foundation \\ *Corresponding author E-mail: chakravarthiparuchuri@gmail.com
}

\begin{abstract}
The expected take-up of Cloud, based on settled research in Web administrations, systems, utility figuring, disseminated processing and virtualization, will get many favorable circumstances cost, changeability and approachability for benefit clients. These advantages are foreseen to propel drive the requirements for Cloud organizations, growing both the Cloud's customer base and the extent of Cloud foundations. This has implications for many technical issues in Service Oriented Architectures and Internet of Services (IoS)-type applications; including fault tolerance, large accessibility and adaptability. Focal to these issues will be the stronghold for successful balancing load techniques. Balancing load includes allocating assignments to every processor and limiting the time for the execution program. Practically speaking, it will be conceivable even to execute the applications on any machine of overall distributed frameworks. We are going to talk the possible distributed solutions proposed for balancing load and pros and cons of load balancing.
\end{abstract}

Keywords: Balancing Load; Cloud; Storage in Cloud; Partition; Replication.

\section{Introduction}

Cloud [2] registering will be another computing. It may be giving web assets and internet stockpiling of the user's. It gives each and every one that the information during an easier cosset. Previously, cloud registering client's camwood entry assets constantly through web. They have should pay main to the individuals assets concerning illustration a great deal they utilization. Done cloud registering cloud supplier outsourced every last one of assets to their customer. There would a unique number existing issues over cloud registering. The basic issues may be load adjusting clinched alongside cloud registering. Load adjusting serves on circulate the larger part loads between all last one of hubs. It additionally ensures that each registering asset may be conveyed fairly and efficiently. It serves to keeping bottlenecks of the framework which might happens because of load lopsidedness. It gives secondary fulfillment of the clients. Load adjusting is a moderately new method that gives secondary asset use What's more exceptional reaction time. Cloud registering give acceptable large portions favorable circumstances of the clients.

Load adjusting calculations need aid ordered as static also progressive calculations. Static calculations would most suitableness to homogeneous and stable situations and might handle exceptionally great brings about these situations.

Changing calculations are additional adaptable Also make under thought different sorts from claiming qualities in the framework both former should What's more throughout run-time. These calculations camwood adjust on transforms what's more provide better brings about heterogeneous Furthermore dynamic situations. In paper we exhibit those overview on the different load adjusting calculations formed Also right now existed on suit of reinforcement those cloud registering situations. We would setting off will provide the over perspective though these calculations and talk about their properties What's more tests for load adjusting On cloud registering nature's domain.

\section{Cloud consist of several characteristics}

\subsection{On demand service}

Cloud [2] provides services to clients on their Layers of Services demand. Users can avail the services as they need and whenever they want.

\subsection{Broad network access}

In cloud capabilities are made available through the network. All the resources are utilized through various methods.

\subsection{Resource pooling}

Various models are used to pool the resources which are provide by the providers to their clients. All the resources dynamically allocate and reallocates according to consumer needs.

\subsection{Rapid elasticity}

Requirement of resources may vary at any time as per the clients or users requirements.

\subsection{Measured service}

In cloud [2] capabilities usage can be measured, controlled for both the service provider and client of the all services provided.

\section{Challenges faced in cloud}

1) Security

2) Efficient balancing of load

3) Monitoring of Performance 
4) Scheduling of Resource

5) Qos management and Scale

6) Requires fast and unintrepreted internet connection

\section{Cloud model}

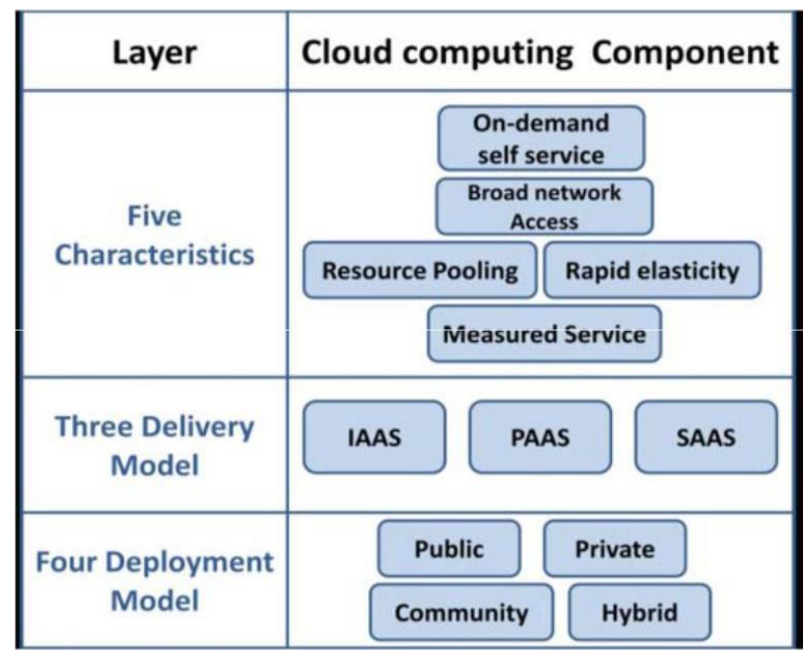

Fig. 1: Model of Cloud Computing.

\section{Services provided by cloud}

Service means various types of functions provided by various servers over the cloud. Many services are providing to the users over cloud.

\subsection{Software as a service (SaaS)}

SaaS provided all the applications to the users which are made available by the providers. Applications are working on a cloud platform. Interfaces (web browser) are used to run the applications. The user does not control the internal function.

\subsection{Platform as a service (PaaS)}

PaaS provide all the capabilities to the users that are required for building applications. It made available all the services through the internet. User need not to download and install the software. Users deploy all the applications onto the cloud infrastructure. There is different languages and tools are provided to the user to develop the applications.

\subsection{Infrastructure as a service (IaaS)}

In this service client does not control or manage the underlying infrastructure of the cloud. In infrastructure as a service, clients are able to control, storage, operating systems and all applications which they deployed. There is a limited control of customer on the networking components.

\section{Layers of services}

All the services have number of layers. Which manage by the consumers and providers.

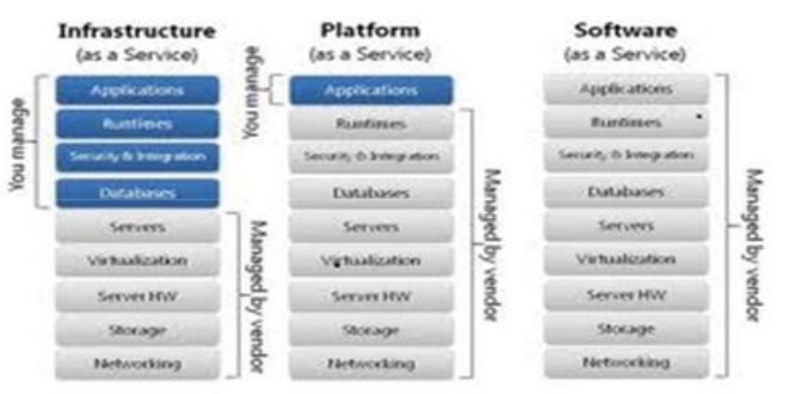

Fig. 2: Layers of Services.

\section{Cloud deployment models}

\subsection{Public cloud}

The cloud stage will be aggravated accessible of the state funded alternately an expansive business gathering furthermore will be claimed by an association. Anybody might utilization open cloud Likewise they have any desire without confinement.

\subsection{Private cloud}

The cloud platform is used by a single organization. Private cloud is only managed by the organization or a third party. Public not able to use the private cloud directly.

\subsection{Community cloud}

The cloud [1] infrastructure is shared by many organizations. Community cloud supports a specific community that has shared concerns.

\subsection{Hybrid cloud}

Hybrid cloud is a combination of two or more clouds (private, community, or public).

\section{Balancing of load in cloud computing}

\subsection{Cloud load balancer}

Load balancer in cloud will be a movement conveyance administration to various CVMs. Cloud load balancer camwood augment provision systems outside administration competencies through movement conveyance Furthermore enhance their accessibility by eliminating absolute side of the point of disappointment Those cloud load offset administration virtualizes various CVM assets found in the same locale under a high-performance, highAccessibility provision administration pool by setting An virtual administration location (VIP), Furthermore conveys those organize solicitations starting with those customer of the CVM pool as it were specified Toward the provision.

Cloud load equalization camwood weigh the wellbeing of CVMs in the CVM Pool, What's more naturally disconnect abnormal CVMs, consequently managing those absolute side of the point about disappointment (SPOF) of a single CVM What's more moving forward those general administration ability about provisions. Tenant cloud load parity gives self-management, selftroubleshooting, anti-network strike and other propelled offers to enterprises, communities, e-commerce, amusements Furthermore different client situations.

\section{Components}

A cloud load balancer one assembly that gives administrations regularly comprises of the Emulating components. 


\subsection{Cloud load balancer}

A cloud load balancer occurrence for movement conveyance. VIP (virtual IP): An ip deliver through which the cloud load balancer gives administration of the customer. Backend/Virtual Server: an assembly from claiming cloud load balancer instances on the backend for preparing solicitations.

VPC/Basic network: the generally speaking organize nature's domain. Get solicitations from other servers other than cloud load balancer is conveyed through the cloud load balancer example of the backend CVM for preparing as stated by those connected approaches Also sending tenets.

\section{Working principle}

The load balancer accepts incoming traffic from the client and routes the request to a back-end CVM instance in one or more available zones for processing.

Cloud load parity administration is mostly furnished toward cloud load balancer audience. Those audience may be answerable for observing solicitations on the cloud load balancer instance, delivering approaches will back-end CVMs Furthermore different benefits. By configuring those sending conventions What's more protocol ports for "client-cloud load balancer" Also "cloud load balancer-backend CVM", cloud load balancer might ahead those appeal straightforwardly to the back-end CVM.

It may be prescribed that you design a back-end CVM occurrence of the cloud load balancer crosswise over numerous accessible zones. Whether an accessible range gets unavailable, those cloud load balancer routes those movement should different accessible zones to typical operation, thereby protecting off the administration interference created toward a solitary accessible zone disappointment.

\section{Load balancing techniques}

1) Carton

2) Compare and Balance

3) Events Driven

4) Scheduling strategy on LB of VM resource

5) Honeybee foraging behavior

6) Biased Random Sampling

7) Message Oriented Model

8) Open Flow Model

9) Self-Organized Load Balancing Algorithm

\section{Metrics for load balancing}

Various metrics considered in existing load balancing techniques [9] in cloud computing are discussed below-

10.1. Scalability is the capacity from claiming a algorithm should perform load adjusting for an arrangement for At whatever limited number for hubs. This metric if make progressed for productive load adjusting.

10.2. Resource Utilization is used to check those use about resources. It ought to further bolstering make optimized to a proficient load adjusting.

10.3. Performance is used to check the effectiveness of the framework. This need should make progressed during a sensible cost, etc., diminish errand reaction time same time keeping satisfactory postponements.

10.4. Response Time is the measure about run through taken with react Eventually Tom's perusing a specific load adjusting algorithm for a conveyed framework. This parameter ought to a chance to be minimized.

10.5. Overhead Associated determines the measure about overhead included same time actualizing a load-balancing algorithm [3]. It may be made from claiming overhead because of development about tasks, inter-processor and bury various trust transform correspondence. This if be minimized along these lines that a load adjusting method might worth of effort proficiently.

\section{Various load balancing algorithms}

1) Round Robin Algorithm

2) Opportunistic load balancing algorithm

3) Minimum completion time

4) Minimum execution time

5) Throttled load balancing algorithm

6) Equally spread current execution algorithm

7) Min Min algorithm

8) Max min algorithm

9) K. Weighted Active Monitoring Load Balancing Algorithm

\subsection{Round robin algorithm}

It will be an undertaking planning strategy on which errands are scheduled In view of chance quantum. A little unit of time will be called duration of the time cut which may be characterized in this algorithm [1]. It will be also known as time offering framework clinched alongside which every procedure will execute time opening without any necessity. These systems gap those assignment ask for just as on the accessible administration suppliers or assets. Round robin may be a static algorithm [3]. Round-robin planning calculation doesn't provide for extraordinary necessity to that's only the tip of the iceberg critical assignments. This implies a dire appeal doesn't get took care of any speedier over other solicitations to queue. Throughput is low similarly as those huge transform may be holding up the focal preparing unit to execution. In round robin, rise to add up of occasion when will make doled out on each transform for execution, However it camwood baffle the individuals for medium-length errands.

\subsection{Opportunistic load balancing algorithm}

This algorithm [1] recognizes the allocated vocation a about each accessible virtual machine What's more relegate the chose unexecuted undertaking of the accessible virtual machine [8] which need those least load "around every last one of virtual machines. OLB relegate those particular occupation in irregular request without recognizing the desire execution time to those occupation on that virtual machine [8]. Likewise a result, it gives finer load balanced, At it provides for poor Generally speaking fruition occasion when (make span). This calculation will be precise basic Furthermore not difficult should implement, and every virtual machine frequently all the stay with occupied.

\subsection{Minimum completion time}

The base fruition the long run particular occupation planning calculation dispatches those chose vocation of the accessible VM that camwood gatherings give those least fruition run through. Because of this motivation behind there need aid some assignments with be allocated will machines that don't have those base execution the long run to them. Those principle ticket about deciding the base fruition run through in the planning calculation is the processor velocity and the cur-rent load for every VM [5].

Minimum Completion time is calculated according to this equation:

$\mathrm{Ctij}=\mathrm{Etij}+\mathrm{rtj}$

Where, $\mathrm{rtj}=$ Time for ready resource $\mathrm{Rj}$.

Etij $=$ Time for execution task Ti on resource $\mathrm{Rj}$.

$\mathrm{Ctij}=$ Time for completion task $\mathrm{Ti}$ on resource $\mathrm{Rj}$. 


\subsection{Throttled load balancing algorithm}

Throttled load adjusting calculation supports the list rundown to the greater part virtual machines [8]. List rundown support those data of the state (Busy/Ideal) of every virtual machine. The point when another a arrives, throttled load balancer examine those indexed rundown starting with the top banana until those Initially allowed virtual machine [8] will be found. Throttled load harmony returns the Perfect virtual machine id al-adha of the information focus controller. It will need queued until those whatever particular case virtual machine gets to be nothing. This calculation is changing.

Major hindrance is that this calculation doesn't Think as of the propel load adjusting necessities like execution duration of the time Furthermore fruition occasion when from claiming each solicitation on the accessible assets for those load adjusting.

\subsection{Equally spread current execution algorithm (ESCE)}

Clinched a long side just as spread current execution method load balancer spread rise to load will every last one of accessible servers or machines virtually which joined with those information focus. Load balancer supports a list table which holds those record about Virtual machines Also number from claiming solicitations right now allocated of the machines virtually (VM). In those demand hails from those information focus will dispense those new VM [5], it filters the list table also fine the any rate stacked virtua machine [8]. Those load balancer likewise comes back the any rate as stacked VM id al-adha of the information core controller. In the event that there would more than you quit offering on that one VM is discovered over initially distinguished VM will be chose to taking care of the solicitation of the client/node. The information focal point revises those list table toward expanding those allotment number of recognized VM. At VM completes the allocated task, a may be communicated to information core which is further notified by those load balancer. The load balancer once more revises those list table by diminishing those allotment number to identifier VM [5] Eventually Tom's perusing one yet all the three will be an extra calculation overhead will examine those queue over and over.

\subsection{Min-min}

Min-Min algorithm [3] begins for a set for unmapped / unscheduled employments. Min-Min assignment planning algorithm will be An static planning calculation. This calculation primary identifies those occupations hosting least execution run through What's more these errands are planned To begin with in this calculation. Afterward it will figure those relied upon fruition period for every assignment as stated by accessible virtual machines [8] and the asset that need those base fruition occasion when for chosen undertaking is planned on that asset. The prepared chance from claiming that asset is updated and the methodology will be repeater until every last one of unexecuted assignments would booked. Subsequently Min-Min algorithm [3] decides the littlest size assignments main Furthermore doled out these errands to speediest rate of asset. Thus it abandons those some asset over-burden and different remains underutilized alternately unmoving pulley. Furthermore it doesn't provide load adjusted in the framework. It will provide an finer make span Also asset usage when the amount of the substantial assignment may be more than those number of the little assignment to meta-task.

Primary hindrance about this calculation will be that it selects little assignments should make executed firstly, which thus extensive errand postponements for a while. Min=Min calculation [3] is fizzled with use assets effectively which prompt a load lopsidedness. Expected completion time of each task to the available resources is calculated using this equation:
$\mathrm{Ctij}=\mathrm{Etij}+\mathrm{rtj}$

Where, $\mathrm{rtj}=$ Time for ready resource $\mathrm{Rj}$

Etij $=$ Time for executing task $\mathrm{Ti}$ on resource $\mathrm{Rj}$

$\mathrm{Ctij}=$ Time for completion task $\mathrm{Ti}$ on resource $\mathrm{Rj}$

\subsection{Max-min}

Max-min calculation [3] allocates undertaking ti on the asset $\mathrm{Rj}$ the place extensive errands bring most astounding necessity as opposed littler errands in the meta-task. It will be start with a set for unmapped errands. Afterward figure needed execution run through Further more needed fruition period of every undertaking with respect to accessible asset. At this point select those undertaking for general greatest fruition the long run Furthermore relegate this errand of the asset which provide for generally least execution duration of the time. The primed the long haul from claiming that asset may be updated. Finally, those new mapped undertaking may be uprooted from those meta-task, and the transform continues rehashing decimal until the sum errands would mapped which intimates till meta-task is void. Those clue about Max-Min calculation may be to decrease those holding up chance of extensive errands. Likewise Max-min provides for most astounding necessity will in length tasks, it builds Normal reaction time to little assignments. This calculation performs exceptional the point when those number about little undertaking may be substantially more than the long person.

Example:

Table 4: Describes the List of Tasks and List of Available Resources.

\begin{tabular}{llll}
\hline Resource & MIPS & Task & MI \\
\hline R1 & 10 & T1 & 10 \\
R2 & 8 & T2 & 15 \\
R3 & 5 & T3 & 20 \\
& & T4 & 25 \\
& & T5 & 50 \\
\hline
\end{tabular}

Table 5: Resource and Task Specification

\begin{tabular}{llll}
\hline Task/Resource & $\mathrm{R} 1$ & $\mathrm{R} 2$ & $\mathrm{R} 3$ \\
\hline T1 & 1 & 1.25 & 2 \\
T2 & 1.5 & 1.875 & 3 \\
T3 & 2 & 2.5 & 4 \\
T4 & 2.5 & 3.12 & 5 \\
T5 & 5 & 6.25 & 10 \\
\hline
\end{tabular}

\subsection{Weighted active monitoring load balancing algo-} rithm

Those Weighted active observing load adjusting calculation may be suggested toward modifying the dynamic following load balancer by relegating a weight with each Virtual machine. In this suggested load adjusting algorithm [3] every one VM would allocated diverse sum of the accessible transforming force. To start with make virtual machines [8] about different registering energy As far as its center processor, preparing speed (MIPS), memory, stockpiling. Dispense weighted number as stated by registering force of virtual machines. Though particular case VM may be skilled about Hosting Double Similarly as significantly load concerning illustration those other, that point doled out weight may be 2 alternately though it might detract four times load then allocated weight is 4 etc.

In this algorithm [1] Weighted Active Vm Load Balancer supports a list table about VMs, connected weighted check and the amount from claiming solicitations at present allocated of the VM [5]. At An appeal will arrive, load balancer parse the list Also Figure the minimum stacked virtual machine [8]. It dispense of a greater part capable VM as stated by the weight doled out. On there are more than one, those to begin with identifier may be chose. Then afterward completing transforming appeal on the virtual machine, load balancer de allocate those virtual machine Furthermore upgrade the asset allotment table Eventually Tom's perusing diminishing allotment number by you quit offering on that one. 


\subsection{Modified throttled algorithm}

Throttled algorithm [1] will be changed for adjusting the load the middle of those assets. To throttled calculation at whatever point the new demand will be land at the cloudlet, those undertaking will be submitted of the throttled virtual machine [8] balancer. In there are more than you quit offering on that one ask for or additional appeal aggregation would introduce At that point it will store under the queue until the a standout amongst virtual machine turns into accessible. For throttled algorithm [3] load balancer uphold record of state (Busy/Available) for virtual machine. When those appeal will be come, load balancer parse the list from those start Also come back those nothing virtual machine [8] id al-adha. In this calculation each time it will parse those list from the start In this way introductory virtual machines need aid vigorously stacked What's more remaining need aid Perfect or under stacked. Throttled algorithm provide for exceptional reaction time be that as it is neglected done relegating every last bit approaching employments uniformly. Altered throttled algorithm defeats know Hindrances of the throttled algorithm. Distinction between the changed throttled algorithm [1] also throttled algorithms is that altered throttled calculation won't parse those list starting with those start every period. At those next appeal arrives, the Virtual machine during list following with now doled out VM [5] will be decided relying upon those state for VM. For suggested algorithm [3] every last one of VMs are used totally what's more appropriately. Suggested calculation conveys load almost uniform Around VMs, with enhanced reaction time contrasted with existing calculations.

\section{Conclusion}

In this study, The conduct about this load adjusting algorithms, namely: round Rubin, Max-Min, Min-Min, ESCE, AMLB, Throttled Algorithm, altered Throttled Algorithm, Weighted dynamic following Algorithm, MET, MCT, enhanced Max-Min, OLB, enhanced expense built calculations need aid analyzed to An cloud registering earth. This load adjusting calculations are assessed by recognizing those significant aspects of the cloud registering surroundings. A portion of the calculations would valuable done a few instances in max-min will be furnish better execution in there need aid amount from claiming little assignments i more than those more ones. There will be not a solitary algorithm [1] that gives greatest throughput, least reaction time What's more successful asset use.

\section{Acknowledgement}

This is a text of acknowledgements. Do not forget people who have assisted you on your work. Do not exaggerate with thanks. If your work has been paid by a Grant, mention the Grant name and number here.

\section{References}

[1] Vishrut Majmudar, Harshal Trivedi and Jitendra Bhatia, "HTV Dynamic Load Balancing Algorithm for Virtual Machine Instances in Cloud" 978-0-7695-4931-6/12 \$26.00 @ 2012 IEEE.

[2] Mishra, Ratan, and Anant Jaiswal. "Ant colony optimization: A solution of load balancing in cloud." International Journal of Web \& Semantic Technology 3.2 (2012): 33.

[3] Gbola akanmu, Professor Frank Wang, Huankai Chen, "UserPriority Guided Min-Min Scheduling Algorithm For Load Balancing in Cloud Computing”, IEEE 2013.

[4] Shu-Ching Wang, Kuo-Qin Yan, Wen-Pin Liao and Shun-Sheng Wang, "Towards a Load Balancing in a Three-level Cloud Computing Network", 978-1-4244-5540-9/10/\$26.00 @2010 IEEE.

[5] Ms. G. Vidya, Mr. M. Ajit, "VM Level Load Balancing in Cloud Environment”, 4th ICCCNT 2013 July 4-6, 2013, Tiruchengode, India Published, IEEE-31661.
[6] Kaur, Rajwinder, and Pawan Luthra. "Load balancing in cloud computing." In Proceedings of International Conference on Recent Trends in Information, Telecommunication and Computing, ITC. 2012.

[7] Chaczko Z, Mahadevan V, Aslanzadeh S, Mcdermid C. Availability and load balancing in cloud computing. In International Conference on Computer and Software Modeling, Singapore 2011 Sep 16 (Vol. 14).

[8] Schmidt M, Fallenbeck N, Smith M, Freisleben B. Efficient distribution of virtual machines for cloud computing. InParallel, Distributed and Network-Based Processing (PDP), 2010 18th Euromicro International Conference on 2010 Feb 17 (pp. 567-574). IEEE. https://doi.org/10.1109/PDP.2010.39.

[9] Bryhni, H., Klovning, E. and Kure, O., 2000. A comparison of load balancing techniques for scalable web servers. IEEE network, 14(4), pp.58-64. https://doi.org/10.1109/65.855480. 